Article

\title{
A 25.78-Gbit/s $\times$ 4-ch Active Optical Cable with Ultra-Compact Form Factor for High-Density Optical Interconnects
}

\author{
Naohiro Kohmu * (D), Toshiaki Takai, Norio Chujo and Hideo Arimoto \\ Hitachi, Ltd. Research \& Development Group; Tokyo 185-8601, Japan; toshiaki.takai.vg@hitachi.com (T.T.); \\ norio.chujo.fj@hitachi.com (N.C.); hideo.arimoto.zh@hitachi.com (H.A.) \\ * Correspondence: naohiro.kohmu.vw@hitachi.com; Tel.: +81-42-323-1111
}

Received: 30 October 2017; Accepted: 17 January 2018; Published: 18 January 2018

\begin{abstract}
A 25.78-Gbit/s $\times$ 4-ch active optical cable (AOC) with an ultra-compact form factor is proposed. The size of the proposed AOC is $5.2 \mathrm{~cm}^{3}$, which is $55 \%$ smaller than the standard form factor of Quad Small Form-factor Pluggable (QSFP28), and 45\% smaller than that of Micro Quad Small Form-factor Pluggable ( $\mu \mathrm{QSFP})$. As a result of utilizing a high-efficiency heat-dissipation structure and optimizing signal transmission lines and ground vias, the proposed AOC achieves high-heat dissipation and low-crosstalk characteristics. Furthermore, the proposed AOC demonstrated 25.78-Gbit/s error-free optical transmission over a 100-m Optical Multimode 3 (OM3) multimode fiber under all-channels (4-ch) operation and case temperature $\left(T_{\mathrm{c}}\right)$ of $70{ }^{\circ} \mathrm{C}$.
\end{abstract}

Keywords: optical interconnect; active optical cable; compact form factor; high-heat dissipation structure; low crosstalk characteristics

\section{Introduction}

Recently, with the drastic increase of data traffic in datacenters (DCs) and the evolution of high-performance computing (HPC), high-speed ( $>25 \mathrm{Gbit} / \mathrm{s}$ ) data communication are required. In the case of conventional electrical interconnects, high-speed and long distance $(>10 \mathrm{~m})$ transmission is challenging due to large transmission loss [1]. On the other hand, optical interconnects have the advantage that high-speed signals can be transmitted further than in an electrical one with low propagation loss. Therefore, as the optical interconnects for rack-to-rack data transmission, active optical cables (AOCs) have been developed and widely applied [2-6]. More recently, for high-density signal transmission, it has become necessary to increase the total input/output (I/O) capacity. To meet that need, we previously proposed a $25.78-\mathrm{Gbit} / \mathrm{s} \times 12$-ch AOC in the same size as the form factor of QSFP28 [7]. However, to meet system requirements, 4-ch and 1-ch AOCs are also required.

To achieve high density signal transmission with 4-ch AOCs, it is necessary to reduce the form-factor of AOCs compared with our previous form-factor (12-ch AOC) or the standard form factor of QSFP28 [7,8]. Miniaturizing the form-factor of an AOC, and therefore reducing its surface area, reduces its heat dissipation efficiency. In addition, miniaturizing the form factor also increases crosstalk noise because high-speed signal transmission lines are fabricated closely together. Therefore, to realize 4-ch AOCs with compact form factor, techniques to achieve higher-heat dissipation and lower inter-channel crosstalk have to be improved.

In this study, we developed a 25.78-Gbit/s $\times 4$-ch AOC integrated with a newly ultra-compact form factor. The proposed AOC has a small size of $5.2 \mathrm{~cm}^{3}$ as a result of applying newly developed high-density packaging techniques, namely, a $0.5-\mathrm{mm}$ pitch electrical connector and high-heat dissipation structure. Furthermore, optimizing signal transmission lines and ground vias improved 8inter-channel crosstalk characteristics. In our experiments, the proposed AOC demonstrated 
25.78-Gbit/s error-free optical transmission under all-channels (4-ch) operation at high temperature (case temperature: $T_{\mathrm{C}}=70^{\circ} \mathrm{C}$ ).

\section{Structure of Proposed AOC}

Figure 1 shows the structure of the proposed AOC. By utilizing a 0.5-mm-pitch electrical connector, which has a 38-pin, and high-density optical assembly technique, the size of the proposed form-factor is $5.2 \mathrm{~cm}^{3}(10.9 \times 56.6 \times 8.4 \mathrm{~mm})$, which is $55 \%$ smaller than that of the standard form-factor of "QSFP28" $\left(12.5 \mathrm{~cm}^{3}: 18.4 \times 80.3 \times 8.5 \mathrm{~mm}\right)$ [8], and $45 \%$ smaller than "MQSFP" $\left(9.4 \mathrm{~cm}^{3}\right.$ : $13.8 \times 64.9 \times 10.6 \mathrm{~mm}$ ) [9]. A cross-sectional view of the proposed AOC is shown in Figure $1 \mathrm{~b}$. In the AOC, a 4-ch vertical cavity surface emitting laser (VCSEL), a 4-ch photodiode (PD), a VCSEL driver integrated circuit (IC), and a transimpedance amplifier (TIA) IC are mounted on the backside of a multi-layer organic substrate. To control the VCSEL driver and the TIA, a micro control unit and an electrically erasable programmable read-only memory (EEPROM) are mounted on the topside of the substrate. An optical connector is placed under the VCSEL and the PD. Thermal vias are fabricated through the substrate to the bottom of the ICs.

(a)

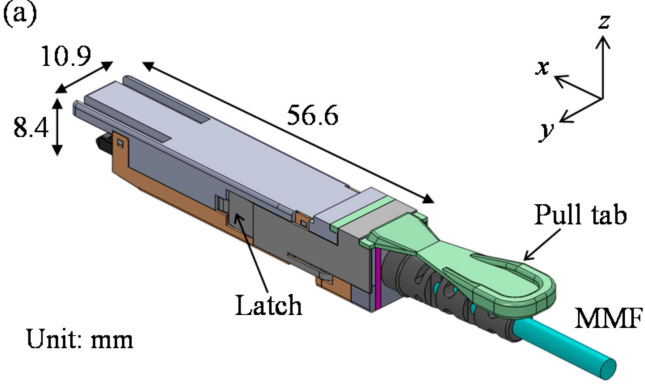

(b)

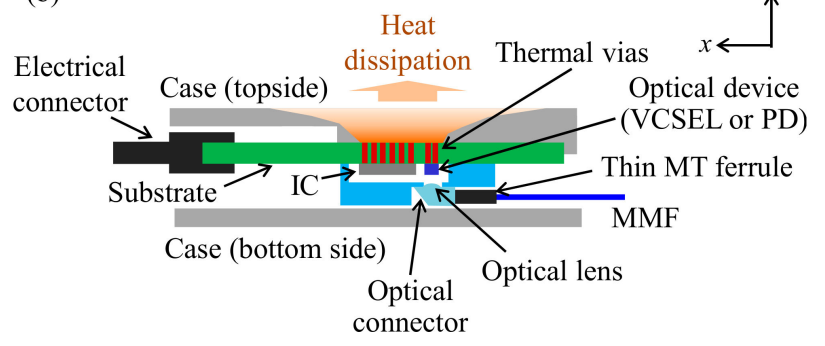

Figure 1. Structure of the proposed active optical cable (AOC) (a) whole view and (b) cross sectional view. MMF, multi mode fiber; IC, integrated circuit; VCSEL, vertical cavity surface emitting laser; PD, photodiode; MCU, micro control unit; EEPROM, electrically erasable programmable read-only memory; MT, mechanical transfer; TIA, transimpedance amplifier.

In the case of a conventional AOC, the ICs (the VCSEL driver and the TIA) are mounted on the top side of the substrate [3-6]. On the contrary, the ICs of the proposed AOC are mounted on the bottom side of the substrate. In the latter structure, as shown in Figure 1b, heat directly dissipates to the topside of the case through the thermal vias. Therefore, compared with the conventional AOCs, the proposed AOC structure can suppress deteriorations in the characteristics of the optical devices in a high-temperature environment.

\section{Design and Analysis}

\subsection{Loss Budget of 25.78-Gbit/s Multimode Optical Link}

To define the target specification of the proposed AOC, a loss budget of a 25.78-Gbit/s multi mode fiber (MMF) optical link was designed. Figure 2 shows the designed loss budget. Transmitter (Tx) optical power was assumed as that of a VCSEL with minimum optical modulation amplitude (OMA) set to $0 \mathrm{dBm}$ under high-temperature operation at VCSEL temperature of $80{ }^{\circ} \mathrm{C}$. In consideration of optical coupling loss caused by beam spreading from the 25-Gbit/s VCSEL, Tx optical-coupling efficiency between the VCSEL and the MMF was set to $-2.0 \mathrm{~dB}$ [7]. Receiver (Rx) optical efficiency between the MMF and the 25-Gbit/s PD was set to $-1.0 \mathrm{~dB}$ [7]. Optical transmission loss of the MMF up to $100 \mathrm{~m}$ (including effects of modal and chromatic dispersions on bit-error-rate (BER) power penalty) was set to $1.5 \mathrm{~dB}$. Moreover, considering the performance of the 25-Gbit/s TIA, optical sensitivity of the Rx side was set to $-6.0 \mathrm{dBm}$ [7]. The link margin was estimated to be $1.0 \mathrm{~dB}$ as shown 
in Figure 2. This margin is assigned to characteristic variations of components and crosstalk penalty under multi-channel operation.

$\underline{\operatorname{Transmitter}(\mathrm{Tx})} \quad \underline{\operatorname{Receiver}(\mathrm{Rx})}$

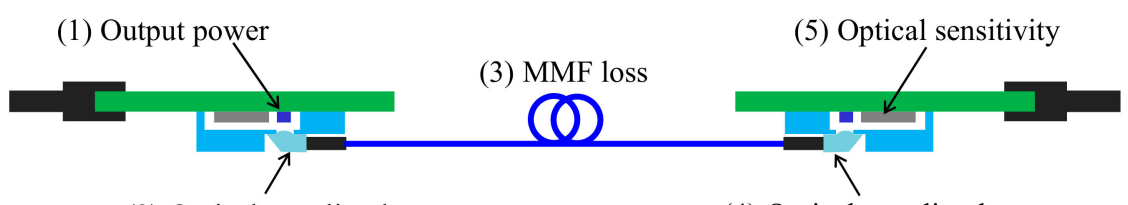

(2) Optical coupling loss

(4) Optical coupling loss

\begin{tabular}{|c|c|c|c|c|c|}
\hline \multicolumn{2}{|c|}{ Tx part } & MMF & \multicolumn{2}{|c|}{ Rx part } & \multirow[b]{2}{*}{$\begin{array}{c}\text { Margir } \\
(\mathrm{dB})\end{array}$} \\
\hline $\begin{array}{l}\text { (1) Output power } \\
\text { (OMA) } \\
(\mathrm{dBm})\end{array}$ & $\begin{array}{l}\text { (2) Optical } \\
\text { coupling loss } \\
\text { (dB) }\end{array}$ & $\begin{array}{l}\text { (3) Loss } \\
\text { (dB) }\end{array}$ & $\begin{array}{l}\text { (4) Optical } \\
\text { coupling loss } \\
\text { (dB) }\end{array}$ & $\begin{array}{c}\text { (5) Optical } \\
\text { sensitivity (OMA) } \\
(\mathrm{dBm})\end{array}$ & \\
\hline 0 & -2.5 & -1.5 & -1.0 & -6.0 & 1.0 \\
\hline
\end{tabular}

Figure 2. Loss budget of 25.78-Gbit/s MMF optical link.

\subsection{Thermal-Via Structure}

To assure the proposed AOC has high heat-dissipation efficiency, the structure of the thermal vias was designed and optimized. Figure 3 shows the cross-sectional view of the thermal vias in a multi-layer substrate. The multi-layer substrate consists of eight organic layers with a total thickness of $0.8 \mathrm{~mm}$. Laser via holes (LVHs) and interstitial via holes (IVHs) are located in the build-up layers (with thickness of $0.05 \mathrm{~mm} /$ layer) and the core substrate (with thickness of $0.5 \mathrm{~mm}$ ), respectively. The LVHs are fabricated by laser-beam machining and filled with copper. The IVHs are fabricated by drilling, and their side walls are formed by copper plating and filled with resin. In addition, a thermal sheet is used to fill in the gap between the substrate and the case. By applying and optimizing this heat-dissipation structure, heat directly and efficiently dissipates to the topside of the case through the thermal vias as shown in Figure 3. Therefore, the proposed AOC allows the optical devices to operate higher temperate.

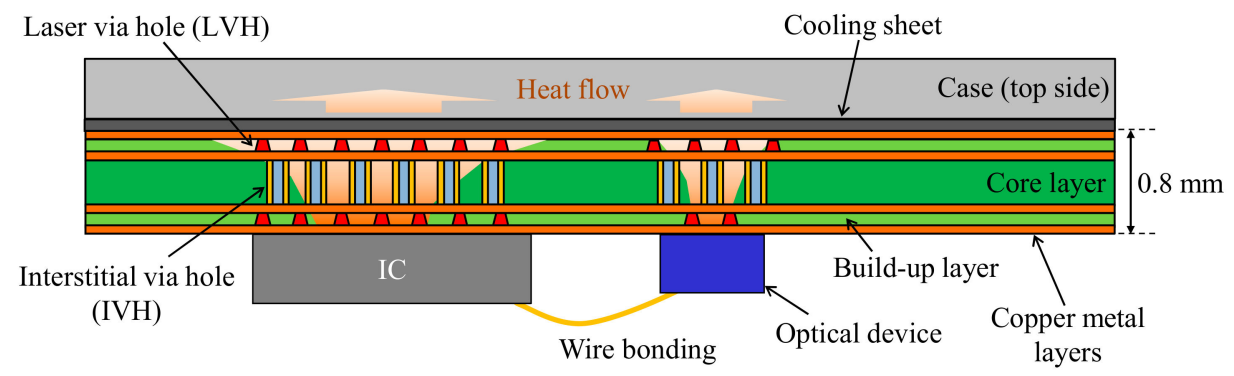

Figure 3. Structure of thermal vias in multi-layer substrate.

\subsection{Inter-Channel Crosstalk}

In regard to parallel optical modules, to suppress influence of inter-channel crosstalk, it is important to optimize the design of signal transmission lines and ground vias structures. Figure $4 \mathrm{a}$ shows the simulation model to analyze crosstalk by using high-speed communication analysis tool, Keysight ADS. Victim and aggressor lanes are coupled by capacitors, $C_{x}$. Crosstalk induced jitter is simulated by changing the capacitance of $C_{x}$. Calculated relationship between inter-channel crosstalk jitter and insertion-loss-to-crosstalk ratio (ICR) is plotted in Figure $4 \mathrm{~b}$. The ICR was defined as the ratio 
of the crosstalk and the insertion loss. The result shows that crosstalk jitter can be almost completely suppressed to less than around 0.01 unit interval (UI) when ICR is small of $<-35 \mathrm{~dB}$.

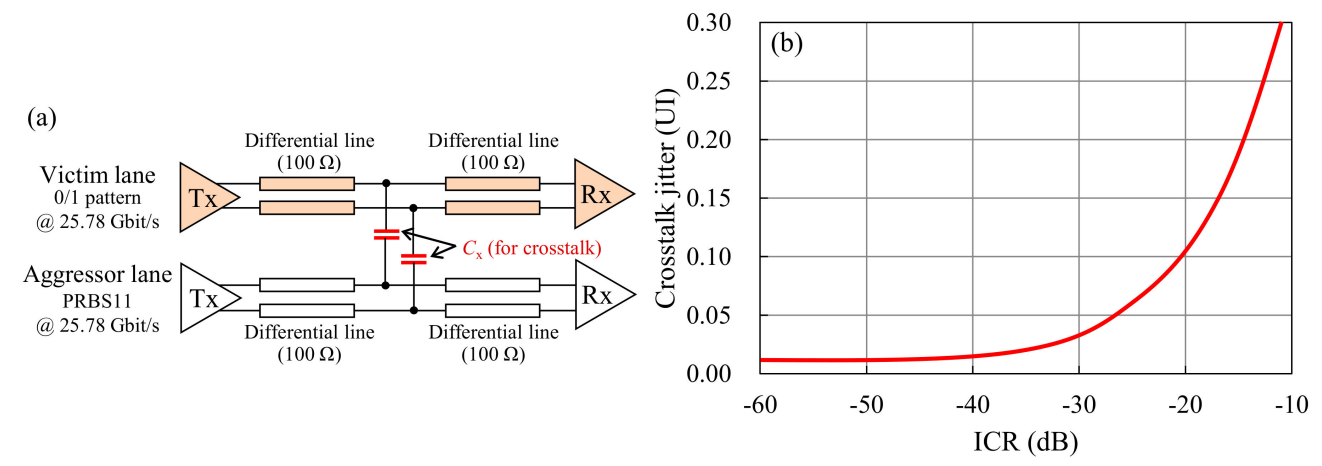

Figure 4. (a) Simulation model for crosstalk analysis and (b) Calculation result of crosstalk jitter. PRBS, pseudo-random binary sequence.

\section{Fabrication}

Based on the design described in Section 3, a prototype of the proposed AOC was fabricated according to the fabrication procedure, shown in Figure $5 \mathrm{a}-\mathrm{d}$, simple assembly technique: First, as shown in Figure 5a, optical devices and ICs were mounted on the backside of the substrate by die and wire bonding. The other ICs (micro control unit (MCU) and EEPROM) were mounted on the topside of the substrate. Second, the optical connector and receptacle was aligned above the optical devices by active alignment method. The optical connector consists of 12-ch optical lenses with a pitch of $250 \mu \mathrm{m}$ and a 12-ch MMF with a 1-mm-thick mechanical transfer (MT) ferrule, and the 4-ch VCSEL and 4-ch PD are aligned with the lenses, simultaneously. Next, the optical connector was fixed onto the substrate by ultraviolet light cure adhesion, and the used master plug connector was removed from the receptacle. Finally, as shown in Figure 5d, they were attached to the case.

(a) Mounting optical devices and ICs

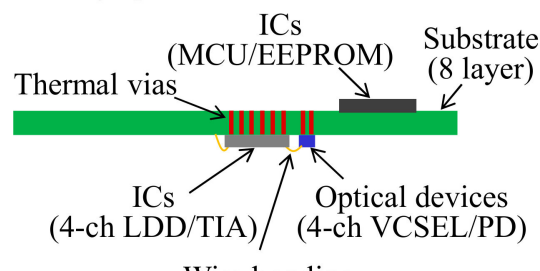

Wire bonding

(c) Adhering receptacle

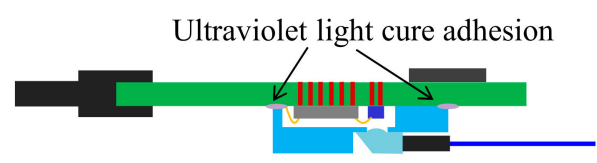

(b) Optical alignment

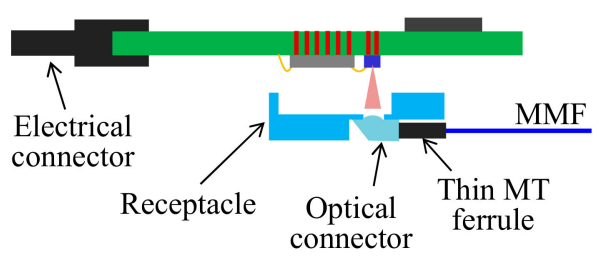

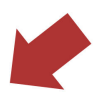

(d) Case attachment

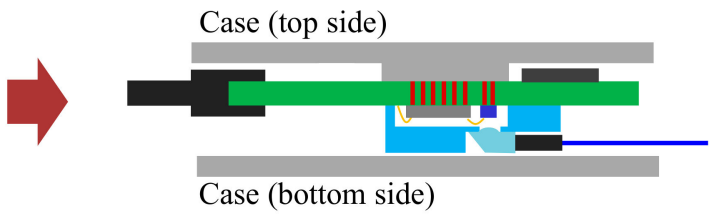

Figure 5. Fabrication procedure of proposed AOC. MCU, micro control unit; LDD, laser diode driver.

Figure 6 shows a photograph of the fabricated AOC. A GaAs- VCSEL and PD, as well as a SiGelaser diode driver (LDD) and TIA with a clock and data recovery (CDR) circuit are adopted as optical devices and ICs, respectively. Total power consumption of the AOC is about $1.6 \mathrm{~W}$ under 4-ch operation with CDR. The breakdown of the AOC's power consumption is about $20 \mathrm{~mW}$ for the VCSEL, $0.7 \mathrm{~W}$ for 
the LDD about $0.8 \mathrm{~W}$ for the TIA and $80 \mathrm{~mW}$ for the control IC (MCU and EEPROM). The MMF used is an $\mathrm{OM} 3$ graded index (GI)-50 $\mu \mathrm{m}$.

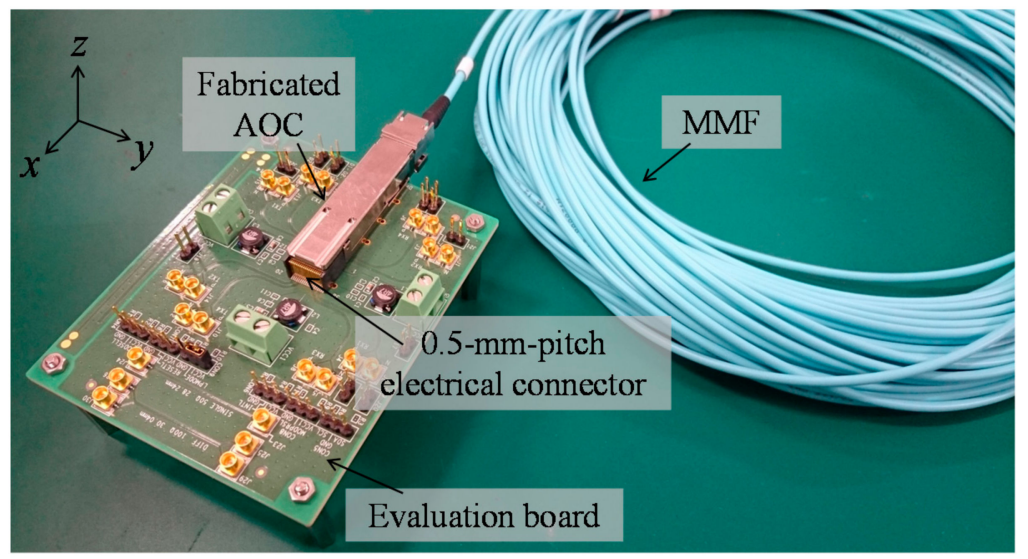

Figure 6. Photograph of fabricated AOC.

\section{Experimental Results}

\subsection{Heat-Dissipation Characteristics}

The temperature of the VCSEL of the fabricated AOC was measured by a thermocouple in a thermostatic chamber with the temperature was controlled so that the case temperature becomes constant at $T_{\mathrm{C}}=70^{\circ} \mathrm{C}$. The measured VCSEL temperature under all-channels (4-ch) operation as shown in Figure 7. This result indicates that the heat-dissipation via structure in the multilayer substrate allows the optical device to operate stably even in a high-temperature environment.

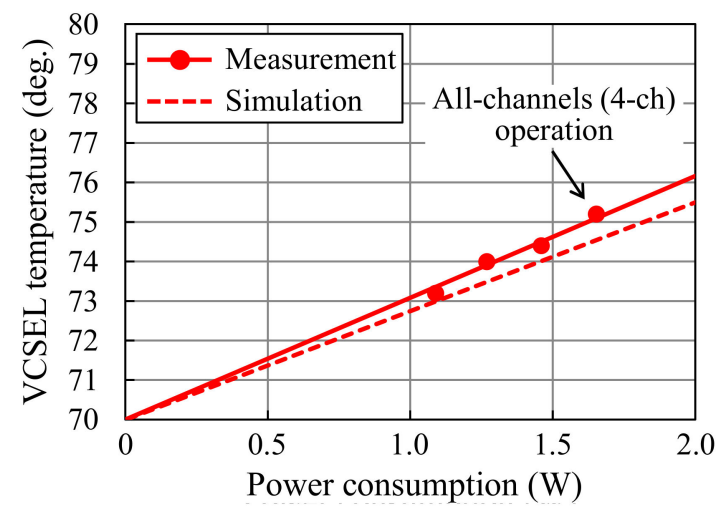

Figure 7. Measured vertical cavity surface emitting laser (VCSEL) temperature.

\subsection{Crosstalk Characteristics}

Signal transmission lines were designed based on the results of the crosstalk analysis in section 3.3. The layout of the signal transmission lines is shown in Figure 8. The pitch of the electrical respectively connector pads is $0.5 \mathrm{~mm}$, width and space of the signal transmission lines are 90 and $120 \mu \mathrm{m}$, respectively. The pitch is smaller than of our previous form-factor AOC [7]. Reduction of the pitch leads to an increase of crosstalk between adjacent channels. Therefore, ground vias were provided along each signal transmission line with no gap for electromagnetic field shielding. Figure 9 shows the measured ICR characteristic of a signal transmission line. The measured ICR at the Nyquist frequency $(12.89 \mathrm{GHz})$ is about $-53 \mathrm{~dB}$. According to the analysis result shown in Figure 4 , that ICR value indicates that crosstalk jitter can be suppressed to less than $0.01 \mathrm{UI}$. 


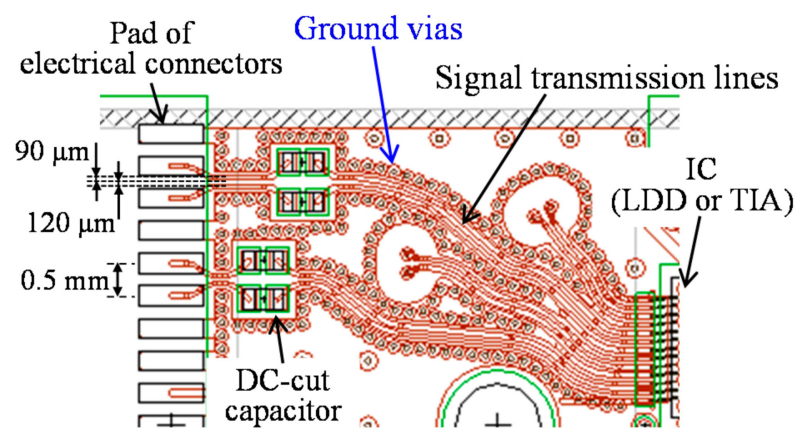

Figure 8. Layout of signal transmission lines and vias.

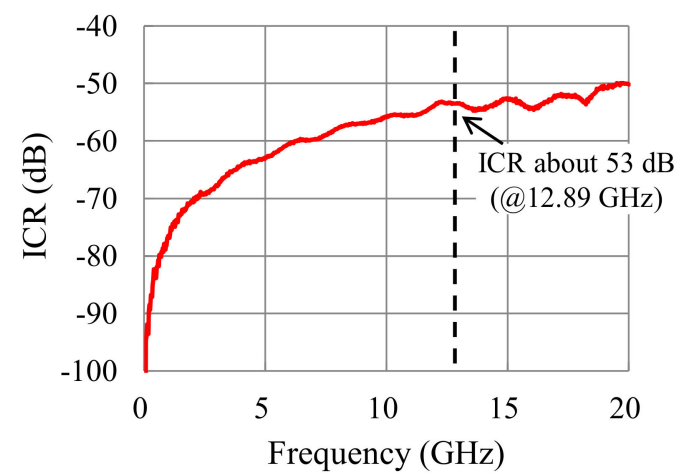

Figure 9. Measured insertion-loss-to-crosstalk ratio (ICR) characteristic.

\subsection{78-Gbit/s Optical Signal Transmission}

Tx optical and Rx electrical waveforms when all channels (4-ch) are active and transmit in loop-back MMF link are shown in Figure 10. Data rate, amplitude, and pattern length of the input electrical signal were set to $25.78 \mathrm{Gbit} / \mathrm{s}, 500 \mathrm{mVppd}$, and $2^{31}-1$ pseudo-random binary sequence (PRBS), respectively. Bias and modulation currents of the VCSEL were set to $6.5 \mathrm{~mA}$ and $8.3 \mathrm{~mA}_{\mathrm{p}-\mathrm{p}}$, respectively. The measured waveforms show clear eye openings for both the transmitter and receiver at room temperature $\left(T_{\mathrm{c}}=25^{\circ} \mathrm{C}\right)$ and high temperature $\left(T_{\mathrm{C}}=70^{\circ} \mathrm{C}\right)$.
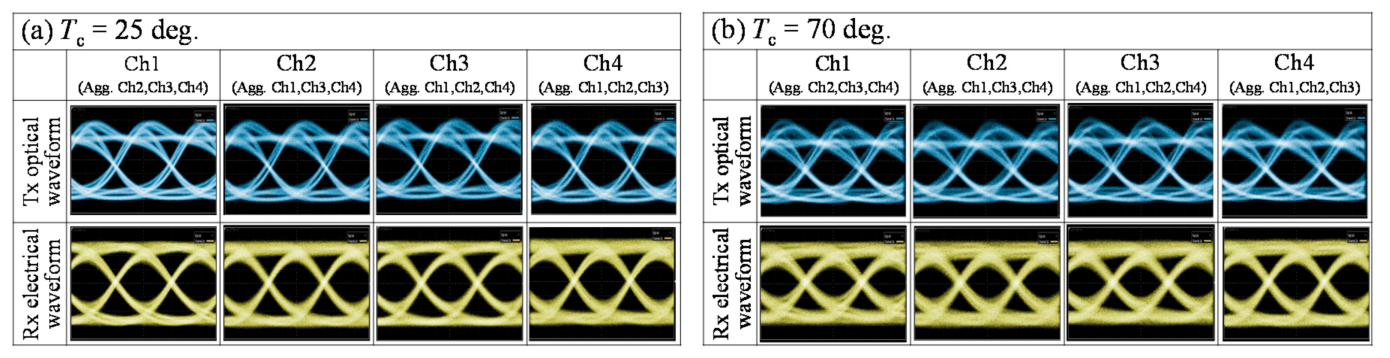

Figure 10. Measured waveforms (a) $T_{\mathrm{C}}=25^{\circ} \mathrm{C}$ and (b) $T_{\mathrm{C}}=70^{\circ} \mathrm{C}$.

Measured bit-error-rate (BER) characteristics for crosstalk are shown in Figure 11. Results show that the measured eye aperture at $\mathrm{BER}=10^{-12}$ is more than $0.60 \mathrm{UI}$ under all channels (4-ch) operation. The measured crosstalk jitter penalty is smaller than 0.03 UI (between 1-ch and 4-ch operations) in good agreement well with the simulation results shown in Figures 4 and 9. 


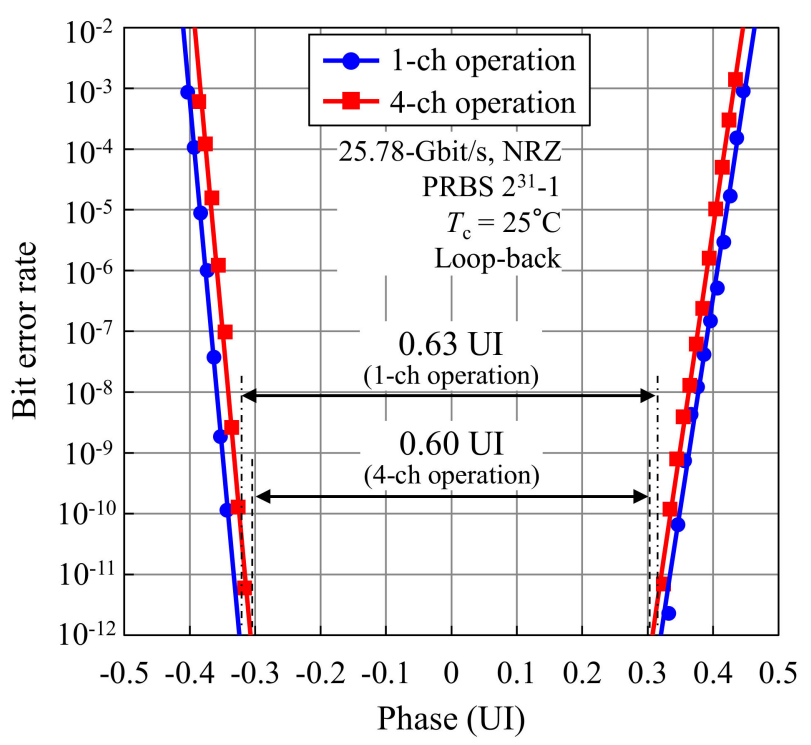

Figure 11. Measured bath-tub curve.

The BER characteristic of the AOC is shown in Figure 12. The result shows that the AOC demonstrated 25.78-Gbit/s error-free transmission under all channels (4-ch) operation and both at $T_{\mathrm{c}}=25$ and $70{ }^{\circ} \mathrm{C}$. According to Figure 12, minimum received optical sensitivities were about $-8.3 \mathrm{dBm}$ at $T_{\mathrm{c}}=25{ }^{\circ} \mathrm{C}$ and about $-9.0 \mathrm{dBm}$ at $T_{\mathrm{c}}=70{ }^{\circ} \mathrm{C}$. The measured BER power penalty is smaller than $0.7 \mathrm{~dB}$ under high temperature $\left(T_{\mathrm{C}}=70^{\circ} \mathrm{C}\right)$. Moreover, the measured BER power penalty less than $1.5 \mathrm{~dB}$ for 100-m MMF link was experimentally demonstrated. Minimum received optical sensitivity was about $-6.9 \mathrm{dBm}$ over 100-m MMF link under all channels operation and high temperature $\left(T_{\mathrm{C}}=70^{\circ} \mathrm{C}\right)$. These results indicate that the proposed AOC, with highly efficient heat dissipation, achieved 25.78-Gbit/s operation.

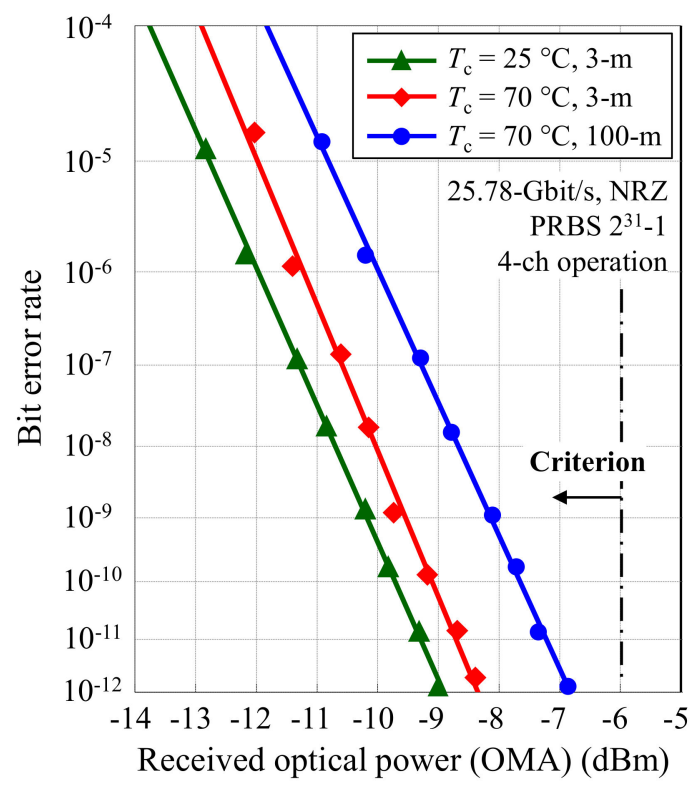

Figure 12. Measured bit-error-rate (BER) characteristics. NRZ, non-return to zero. 


\section{Discussion and Conclusions}

A 25.78-Gbit/s $\times 4$-ch AOC with an ultra-compact form-factor was proposed and fabricated. As a result of using a $0.5-\mathrm{mm}$ pitch electrical connector, the proposed AOC size is $5.2 \mathrm{~cm}^{3}$. In addition, high heat-dissipation structure and signal transmission lines and ground vias were designed and optimized to guarantee 25.78-Gbit/s error-free optical transmission over 100-m MMF link under all-channels (4-ch) operation and high temperature $\left(T_{\mathrm{c}}=70{ }^{\circ} \mathrm{C}\right)$. These results indicate that the proposed AOC is applicable to optical interconnects for short-reach optical communication specified in IEEE802.3 standards.

Table 1 shows a bench mark of form factors for 4-ch AOCs. Our newly-proposed ultra-compact form-factor has the smallest size among the form-factors listed. And its transmission density is higher than those of the other standard form factors. In addition, total interface capacity when the proposed form factor is applied in a 19-inch/1-U rack (in two rows) is $7.2 \mathrm{Tbit} / \mathrm{s}$. Therefore, it is concluded that the proposed AOC can provide high-speed and large-capacity rack-to-rack data communication systems in DCs and HPCs.

Table 1. Benchmark of form-factors for 4-ch active optical cables (AOCs).

\begin{tabular}{ccccc}
\hline Item & Unit & QSFP28 [8] & $\mu$ QSFP [9] & This Work \\
\hline Size & $\mathrm{cm}^{3}$ & 12.5 & 9.4 & 5.2 \\
Transmission density & Gbit/s/cm & 8.2 & 11.0 & 19.8 \\
Total interface capacity (19-inch/1U rack) & Tbit/s & 3.6 & 4.8 & 7.2 \\
\hline
\end{tabular}

Author Contributions: Naohiro Kohmu, Toshiaki Takai, Norio Chujo and Hideo Arimoto conceived and designed the proposed AOC. They performed the experiments, and wrote the paper.

Conflicts of Interest: The authors declare no conflict of interest.

\section{References}

1. Norimatsu, T.; Kawamoto, T.; Kogo, K.; Kohmu, N.; Yuki, F.; Nakajima, N.; Muto, T.; Nasu, J.; Komori, T.; Koba, H.; et al. A $25 \mathrm{~Gb} / \mathrm{s}$ multistandard serial link transceiver for $50 \mathrm{~dB}$-loss copper cable in $28 \mathrm{~nm}$ CMOS. In Proceedings of the IEEE International Solid-State Circuits Conference (ISSCC), San Francisco, CA, USA, 31 January-4 February 2016.

2. Doany, F.E.; Lee, B.G.; Kuchta, D.M.; Rylyakov, A.V.; Baks, C.; Jahnes, C.; Libsch, F.; Schow, C.L. Terabit/sec VCSEL-based parallel optical module based on holey CMOS transceiver IC. J. Lightwave Technol. 2013, 31, 672-680. [CrossRef]

3. Chacinski, M.; Chitica, N.; Molin, S.; Lalic, N.; Sahlén, O. 25.78 Gbps data transmission with $850 \mathrm{~nm}$ multimode VCSEL packaged in QSFP form factor module. In Proceedings of the 2013 Optical Fibre Communications Conference (OFC), Anaheim, CA, USA, 17-21 March 2013. Paper OW1B.1.

4. Tsunoda, Y.; Shibasaki, T.; Oku, H.; Ide, S.; Mori, T.; Koyanagi, Y.; Tanaka, K.; Ishihara, T.; Tamura, H. 25.78-Gb/s VCSEL-based optical transceiver with retimer embedded driver and receiver ICs. In Proceedings of the Optical Fiber Communications Conference (OFC), Los Angeles, CA, USA, 22-26 March 2015. Paper Tu3G.4.

5. Tsunoda, Y.; Shibasaki, T.; Oku, H.; Matsui, J.; Shiraishi, T.; Ide, S.; Mori, T.; Koyanagi, Y.; Tamura, H. 24 to $34-\mathrm{Gb} / \mathrm{s} \times 4$ multi-rate VCSEL-based optical transceiver with referenceless CDR. In Proceedings of the Optical Fiber Communications Conference (OFC), Anaheim, CA, USA, 20-24 March 2016. Paper Th4D.4.

6. Nagashima, K.; Kise, T.; Ishikawa, Y.; Nasu, H. A Record 1-km MMF NRZ 25.78-Gb/s Error-Free Link Using a 1060-nm DIC VCSEL. IEEE Photonics Technol. Lett. 2016, 28, 418-420.

7. Matsuoka, Y.; Kohmu, N.; Lee, Y.; Arimoto, H.; Takai, T.; Chujo, N.; Sato, M.; Komatsuzaki, S.; Ogura, A.; Yamazaki, K.; et al. A compact 310-Gb/s Optical Transceiver for High-Density Optical Interconnects. IEEE Photonics Technol. Lett. 2017, 29, 334-337. [CrossRef] 
8. SFF-8436 Specification for QSFP+ 10 Gbs 4x Pluggable Transceiver. Available online: http:/ /www.optixcom. com/product_pdf/1.duplex/3-QSFP/QSFP_MSA.pdf (accessed on 9 October 2017).

9. MicroQSFP Multi-Source Agreement (MSA). Available online: http://www.microqsfp.com/ (accessed on 9 October 2017).

(․) (1)

(C) 2018 by the authors. Licensee MDPI, Basel, Switzerland. This article is an open access article distributed under the terms and conditions of the Creative Commons Attribution (CC BY) license (http:/ / creativecommons.org/licenses/by/4.0/). 